\title{
Inhibition of ornithine decarboxylase 1 facilitates pegylated arginase treatment in lung adenocarcinoma xenograft models
}

\author{
SZE-KWAN LAM ${ }^{1}$, KIN PONG $\mathrm{U}^{2}$, YUAN-YUAN LI ${ }^{1}$, SHI XU ${ }^{1}$, \\ PAUL NING-MAN CHENG ${ }^{2}$ and JAMES CHUNG-MAN HO ${ }^{1}$ \\ ${ }^{1}$ Division of Respiratory Medicine, Department of Medicine, The University of Hong Kong, \\ Queen Mary Hospital; ${ }^{2}$ Bio-Cancer Treatment International, Hong Kong, SAR, P.R. China
}

Received January 29, 2018; Accepted July 2, 2018

DOI: $10.3892 /$ or.2018.6598

\begin{abstract}
Arginine depletion has shown anticancer effects among arginine auxotrophic cancers. An anti-proliferative effect of pegylated arginase (BCT-100) has been shown in acute myeloid leukaemia, hepatocellular carcinoma and mesothelioma. The aim of the present study was to evaluate the effect of BCT-100 in lung adenocarcinoma. A panel of lung adenocarcinoma cell lines and xenograft models were used to investigate the effect of BCT-100. Protein expression, arginine level, putrescine level, spermidine level and apoptosis were analyzed by western blotting, ELISA, high performance liquid chromatography, dot blot and TUNEL assay, respectively. BCT-100 converts arginine to ornithine. BCT-100 reduced in vitro cell viability across different lung adenocarcinoma cell lines and suppressed tumour growth in an HCC4006 xenograft, while paradoxical growth stimulation was observed in $\mathrm{H} 358$, HCC827, H1650 and H1975 xenografts. Upon BCT-100 treatment, ornithine decarboxylase 1 (ODC1) was induced in two solid tumour xenografts (H1650 and H1975). It was postulated
\end{abstract}

Correspondence to: Dr James Chung-Man Ho, Division of Respiratory Medicine, Department of Medicine, The University of Hong Kong, Queen Mary Hospital, Pokfulam, Hong Kong, SAR, P.R. China

E-mail: jhocm@hku.hk

Abbreviations: ODC1, ornithine decarboxylase 1; DFMO, $\alpha$-difluoromethylornithine; OTC, ornithine transcarbamylase; ASS1, argininosuccinate synthetase; SRM, spermidine synthase; SMS, spermine synthase; SSAT, spermidine/spermine N1-acetyltransferase; HCC, hepatocellular carcinoma; EGFR, epidermal growth factor receptor; ATCC, American Type Culture Collection; MTT, 3-(4,5-dimethylthiazol-2-yl)-2,5-diphenyltetrazolium bromide; HRP, horseradish peroxidase; ECL, enhanced chemiluminescence; PBS, phosphate-buffered saline; OPA, o-phthaldialdehyde; TUNEL, terminal deoxynucleotidyl transferase-dUTP nick end labeling; TdT, terminal deoxynucleotidyl transferase; DAPI, 4',6-diamidino-2-phenylindole

Key words: lung adenocarcinoma, pegylated arginase, ornithine decarboxylase 1 inhibitor, apoptosis, xenograft models that the accumulated ornithine could be channeled via ODC1 to produce polyamines that promoted tumour growth. The action of an ODC1 inhibitor ( $\alpha$-difluoromethylornithine, DFMO) was studied in the restoration of the anticancer effects of BCT-100 in lung adenocarcinoma. In both H1650 and H1975 xenografts, a combination of DFMO and BCT-100 significantly suppressed tumour growth, resulting in doubled median survival compared with the control. Putrescine was decreased in almost all treatment arms in the H1650, H1975 and HCC4006 xenografts. Nonetheless spermidine was reduced only following DFMO/BCT-100 treatment in the H1650 and H1975 xenografts. Apoptosis was enhanced in the combined treatment arm in both $\mathrm{H} 1650$ and $\mathrm{H} 1975$ xenografts. In the HCC4006 xenograft, addition of DFMO did not alter the tumour suppressive effect of BCT-100. In conclusion, inhibition of ODC1 by DFMO was crucial in facilitating BCT-100 treatment in lung adenocarcinoma that was partially mediated by depleting arginine and polyamines with consequent apoptosis.

\section{Introduction}

Amino acids are essential for different cellular functions including synthesis of protein, nitric oxide, urea, creatine and polyamines (1). Thus, amino acid depletion can potentially serve as an effective treatment for cancers.

Arginine is a semi-essential amino acid in some tumours, but non-essential in normal cells. Therefore, arginine-degrading enzymes (arginase and arginine deiminase) have recently been studied in the treatment of different types of cancer that lack the ability to re-synthesize arginine $(2,3)$.

Arginine can be converted to ornithine, citrulline and argininosuccinate by arginase, ornithine transcarbamylase (OTC) and argininosuccinate synthetase (ASS1), respectively. Polyamines (putrescine, spermidine and spermine) are aliphatic cations with pleiotropic functions and are found in various cell types. The biosynthesis of polyamines involves ornithine decarboxylase 1 (ODC1) and S-adenosylmethionine decarboxylase, as well as spermidine synthase (SRM) and spermine synthase (SMS). Nonetheless spermidine/spermine N1-acetyltransferase (SSAT), FAD-dependent polyamine oxidase and spermine oxidase are responsible for the degradation of polyamines (Fig. 1). Polyamines are essential to cell 
proliferation that can also promote tumour growth (4). High expression of ASS1 and OTC, which are the key enzymes in the urea cycle that are responsible for replenishing arginine, is biologically predictive of refractoriness to arginase treatment.

BCT-100 (Bio-Cancer Treatment International Ltd., Hong Kong) is a pegylated (PEG) formulation of arginase (US FDA IND granted in March 2012) that was developed as an anticancer agent. An anti-proliferative effect of BCT-100 has been demonstrated in acute myeloid leukaemia (5), hepatocellular carcinoma (HCC) $(2,6)$ and mesothelioma $(7)$, with an $\mathrm{IC}_{50}$ value of $0.1-1.25,100-250$ and $13-23 \mathrm{mU} / \mathrm{ml}$, respectively. Cell cycle arrest and apoptosis are induced by BCT-100 in human melanoma (8), HCC (9) and mesothelioma (7). BCT-100 has recently demonstrated clinical activity in a phase $\mathrm{I} / \mathrm{II}$ clinical trial in HCC (10). The present study aimed to investigate the role of BCT-100 as an anticancer treatment for lung adenocarcinoma.

\section{Materials and methods}

Cell lines and reagents. A panel of seven lung adenocarcinoma cell lines [epidermal growth factor receptor (EGFR) wild-type and K-ras mutated (H23 and H358) or EGFR mutated (HCC827, H1650, H1975, HCC2935 and HCC4006)] was obtained from The American Type Culture Collection (ATCC, Manassas, VA, USA). The passage of cells used for various experiments was authenticated by ATCC in December 2016 by comparing the ATCC reference database profile. Cells were cultured in Gibco ${ }^{\circledR}$ RPMI-1640 medium (Thermo Fisher Scientific, Inc., Waltham, MA, USA) supplemented with $10 \%$ fetal bovine serum (FBS; Gibco ${ }^{\circledR}$; Thermo Fisher Scientific, Inc.) in a humidified atmosphere of $5 \% \mathrm{CO}_{2}$ at $37^{\circ} \mathrm{C}$.

Pegylated arginase (BCT-100) and $\alpha$-difluoromethylornithine (DFMO). Pegylated arginase (BCT-100, PEG-BCT-100 or rhArglpeg5000) was manufactured and donated by Bio-Cancer Treatment International. DFMO was purchased from Shijiazhuang Aopharm Import \& Export Trading Co., Ltd. (Hebei, China).

Cell viability assay. The 3-(4,5-dimethylthiazol-2-yl)2,5-diphenyltetrazolium bromide (MTT) cell viability assay was performed as previously described (11). Briefly, 5,000 cells were plated in each well and treated for $72 \mathrm{~h}$ with BCT-100. Culture medium alone was served as the control.

Protein expression by western blot analysis and spermidine level by dot blot. Specific primary antibodies [mouse monoclonal anti-human $\beta$-actin (1:1,000; cat. no. A1978) (Sigma-Aldrich; Merck KGaA, Darmstadt, Germany), anti-ASS1 (1:1,000; cat. no. SC-99178), anti-ODC1 (1:1,000; cat. no. SC-33539), anti-OTC (1:1,000; c cat. no. SC-102051), anti-spermidine synthase (SRM) (1:1,000; cat. no. SC-374524), anti-spermine synthase (SMS) (1:1,000; cat. no. SC-99159), anti-cleaved PARP (1:1,000; cat. no. SC-9542) (Santa Cruz Biotechnology, Inc., Santa Cruz, CA, USA), anti-PEG (1:1,000; cat. no. 31-1008-00) (RevMAb Biosciences, San Francisco, CA, USA), anti-survivin (1:1,000; cat. no. 2808), (Cell Signaling Technology, Inc., Danvers, MA, USA)] and corresponding horseradish peroxidase (HRP)-conjugated secondary antibody (anti-mouse IgG, 1:1,000; cat. no. 7076 or anti-rabbit IgG, 1:1,000; cat. no. 7074) (from Cell Signaling Technology, Inc.) were purchased. Western blot analysis was performed as previously reported (12). Cells $\left(5 \times 10^{6}\right)$ were collected and lysed for $1 \mathrm{~h}$ on ice with RIPA buffer (20 mM Tris- $\mathrm{HCl}$ (pH 7.5), $150 \mathrm{mM}$ $\mathrm{NaCl}, 1 \%$ sodium deoxycholate, $1 \mathrm{mM}$ EGTA, $2.5 \mathrm{mM}$ sodium pyrophosphate, $1 \mathrm{mM} \mathrm{Na}{ }_{2}$ EDTA, $1 \% \mathrm{NP}-40,1 \mathrm{mM} \mathrm{Na} \mathrm{VO}_{4}$, $1 \mathrm{mM} \beta$-glycerophosphate and $1 \mu \mathrm{g} / \mathrm{ml}$ leupeptin) including a protease inhibitor cocktail. Tissue samples collected from xenograft models were lysed for $1 \mathrm{~h}$ on ice with T-PER ${ }^{\circledR}$ Tissue Protein Extraction Reagent (cat. no. 78510) (Thermo Fisher Scientific, Inc., Waltham, MA, USA) including a protease inhibitor cocktail. For dot blotting, samples were directly spotted onto a nitrocellulose membrane and allowed to dry for $1 \mathrm{~h}$ at room temperature. The membranes were blocked, incubated with anti-spermidine antibody (1:1,000; cat. no. NB100-1847) (Novus Biologicals, LCC, Littleton, CO, USA) and corresponding secondary antibody (anti-rabbit IgG, 1:1,000; cat. no. 7074; Cell Signaling Technology, Inc.), similar to western blotting. An enhanced chemiluminescence (ECL) kit (GE Healthcare, Buckinghamshire, UK) was used to detect protein expression. $\beta$-actin served as a housekeeping protein.

Tumour xenograft growth in vivo. H358, HCC827, H1650, H1975 and HCC4006 xenografts were established by subcutaneous injection of the corresponding $\left(10^{7}\right)$ cells in phosphate-buffered saline (PBS) into the upper back of 12 (6 mice $\mathrm{x} 2$ groups) or 32 (8 mice $\mathrm{x} 4$ groups) nude mice (female; age, 4- to 6-weeks; weight, 10-14 g, BALB/cAnN-nu; Charles River Laboratories, Wilmington, MA, USA). The mice were kept in 12-h/light/dark cycle with temperature $\left(20-25^{\circ} \mathrm{C}\right)$ and humidity $(60-70 \%)$ control and ad libitum diet was provided. Treatment started when the tumor size reached $\sim 50 \mathrm{~mm}^{3}$. To study the effect of BCT-100, mice were randomized to one of two groups, after tumour growth was established ( $\mathrm{n}=6)$. PBS (control) or BCT-100 $(20 \mathrm{mg} / \mathrm{kg}$ twice a week, intraperitoneally) was administered. To study the combined effect of DFMO and BCT-100, mice were randomized into four groups after tumour growth was established $(\mathrm{n}=8)$. PBS (control), 2\% DFMO (in drinking water), BCT-100 $(20 \mathrm{mg} / \mathrm{kg}$ twice a week, intraperitoneally) or DFMO/BCT-100 was given accordingly. Tumour dimension (using standard calipers) and body weight of mice were assessed twice a week and tumour volume was calculated as follows: Volume $=$ length $\mathrm{x}$ width $\mathrm{x}$ width)/2 (11). For humane reasons, mice were sacrificed (by administration of $100 \mu \mathrm{l}$ pentobarbital sodium solution, intraperitoneally) when tumour size reached $600 \mathrm{~mm}^{3}$. Tumour xenografts were harvested. The study protocol was approved by the institutional Animal Ethics Committee of The University of Hong Kong (approval ref. no. CULATR 3781-15) and standard humane endpoints for animal research were applied in compliance with the instructions of the U.S. Public Health Service (policy on humane care and use of laboratory animals).

Serum arginine concentration. L-arginine ELISA kit was purchased from Immundiagnostik (Bensheim, Hessen, Germany) and the assay was performed according to the manufacturer's protocol. In brief, control, standards and samples were derivatized and incubated with L-arginine antibody overnight. After washing with washing buffer, peroxidase conjugate was added. The reaction was stopped following incubation with 


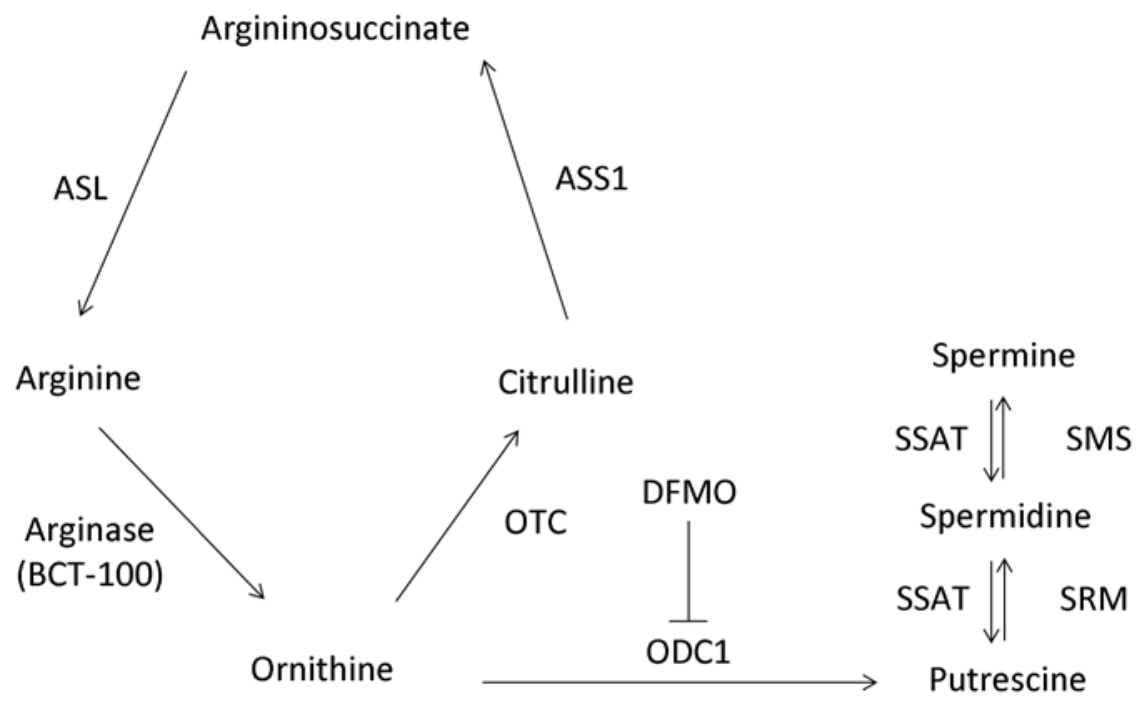

Figure 1. Schematic diagram of the urea cycle and polyamine production. ASS1, argininosuccinate synthetase; OTC, ornithine transcarbamylase; ASL, argininosuccinate lyase; ODC1, ornithine decarboxylase 1; SRM, spermidine synthase; SMS, spermine synthase; SSAT, spermidine/spermine N1-acetyltransferase; DFMO, $\alpha$-difluoromethylornithine.

tetramethybenzidine substrate (13). Absorbance (450 nm) was determined with a reference $(620 \mathrm{~nm})$ using a FLUOstar Optima microplate reader (Bmg Labtec GmbH, Ortenberg, Germany).

Putrescine concentration assessement by high performance liquid chromatography (HPLC). The concentration of putrescine in different tumour lysates was analyzed according to a previously reported methodology (14). Putrescine dihydrochloride and o-phthaldialdehyde (OPA) reagent solution (Sigma-Aldrich; Merck KGaA) and HPLC grade methanol (Tedia Company, Fairfield, OH, USA) were purchased. The standards and samples were centrifuged at $13,400 \mathrm{x}$ g for $10 \mathrm{~min}$. Supernatant $(30 \mu \mathrm{l})$ was mixed with $5 \%$ perchloric acid $(30 \mu \mathrm{l})$ to precipitate proteins. The mixtures were then centrifuged (13,400 x g, $10 \mathrm{~min})$. The supernatant of acidic extract $(50 \mu \mathrm{l})$ was neutralized with borate buffer $(100 \mu \mathrm{l}$, $0.1 \mathrm{M}, \mathrm{pH} 9.0)$ and OPA reagent $(60 \mu \mathrm{l})$ was then added. The derivatized mixtures were centrifuged $(13,400 \mathrm{x} \mathrm{g}, 10 \mathrm{~min})$ and the supernatant $(20 \mu 1)$ was injected into the HPLC system. Nucleosil ODS column (250x4.6 mm, internal diameter $5 \mathrm{~mm}$ ) (Macherey-Nagel GmbH \& Co., Düren, Germany) was connected to Agilent 1260 Infinity (Agilent Technologies, Santa Clara, CA, USA) and eluted with buffer A (water) and buffer B (methanol) at a flow rate of $1 \mathrm{ml} / \mathrm{min}$. Following injection of standards or samples, the column was eluted with $70 \%$ buffer B for $1 \mathrm{~min}$ and an isocratic gradient from $70 \%$ buffer B to $90 \%$ solvent B for 13 min. The column was washed with $100 \%$ buffer B for 5 min and re-equilibrated with $100 \%$ buffer A for $5 \mathrm{~min}$. Signals were detected with an excitation wavelength of $360 \mathrm{~nm}$ and emission wavelength of $510 \mathrm{~nm}$.

Terminal deoxynucleotidyl transferase-dUTP nick end labeling (TUNEL) assay. TUNEL assay was performed using Click-iT $^{\circledR}$ Plus TUNEL assay (Invitrogen; Thermo Fisher Scientific, Inc.). De-paraffinization, fixation and permeabilization of formalin-fixed, paraffin-embedded tumour xenograft sections were performed first. Sections were incubated with

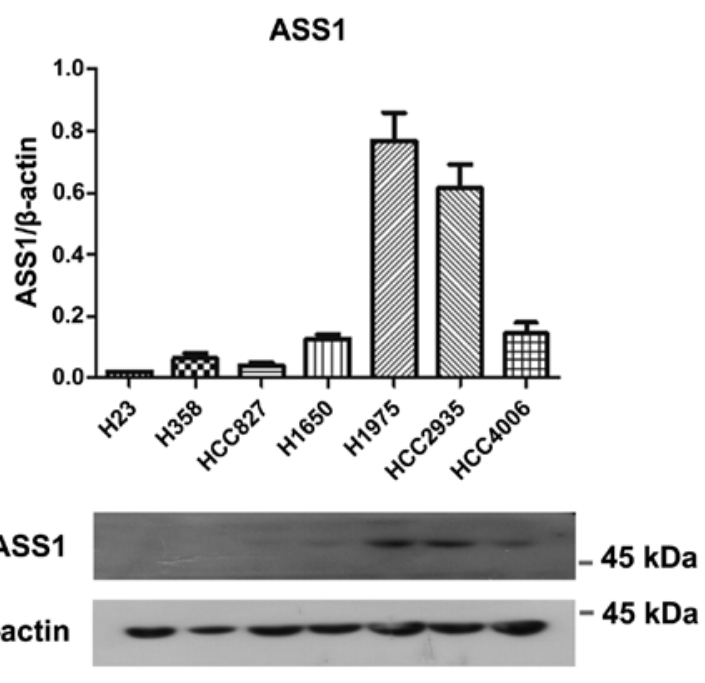

Figure 2. Basal expression of ASS1 in a panel of lung adenocarcinoma cell lines. ASS1 was highly expressed in H1975 and HCC2935 cells.

terminal deoxynucleotidyl transferase (TdT) reaction buffer, and then incubated with TdT buffer containing EdUTP, TdT and TdT enzyme. TUNEL reaction cocktail (Alexa Fluor ${ }^{\circledR}$ picoyl azide, copper protectant, TUNEL reaction buffer additive and TUNEL reaction buffer) was added to each section. The slides were mounted with Prolong ${ }^{\circledR}$ Gold antifade reagent (Invitrogen; Thermo Fisher Scientific, Inc.) containing 4',6-diamidino-2-phenylindole (DAPI). Images were captured using a Nikon Ni-U fluorescence microscope (Nikon, Tokyo, Japan) equipped with a camera/detector Diagnostic Instrument RT3 slider (Meyer Instruments, Houston, TX, USA). Images were captured at x400 magnification using CFI Plan Fluor DLL 40X objective (Nikon). Images were captured using NIS-Elements Basic Research software (SPOT ${ }^{\mathrm{TM}}$ Software 5.0) (Laboratory Imaging Ltd., Prague, Czech Republic).

Statistical analysis. Experiments were repeated at least three times and data were analysed (mean \pm standard error of the 
A

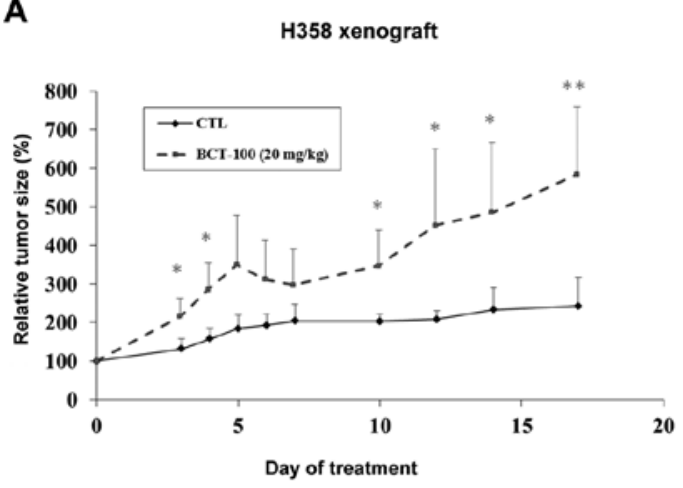

HCC827 xenograft

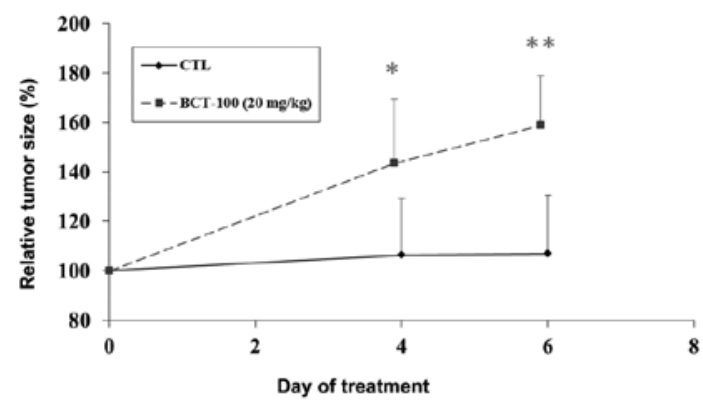

H1650 xenograft

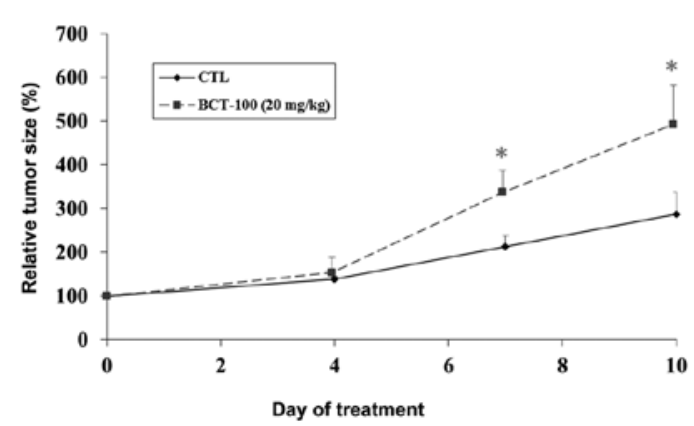

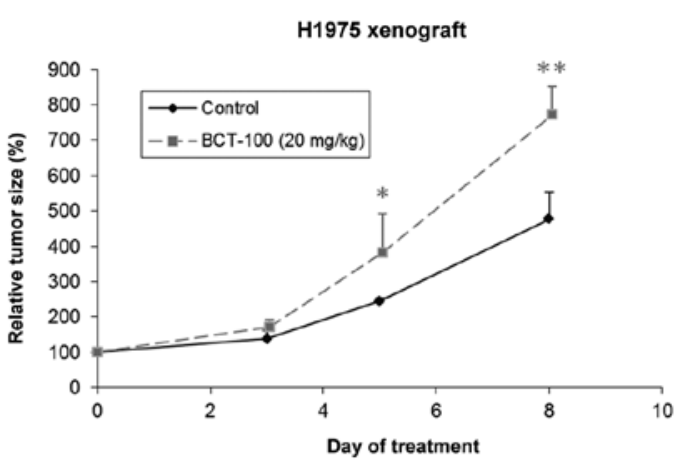

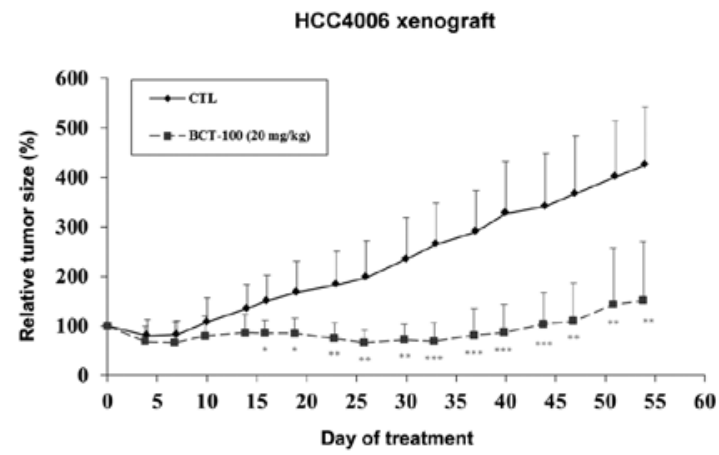

Figure 3. Alteration of tumour size as well as ASS1, OTC, ODC1, SRM and SMS expression in xenograft models following treatment with BCT-100. (A) BCT-100 (dotted lines) stimulated tumour growth in H358, HCC827, H1650 and H1975 xenograft models but inhibited growth in HCC4006 xenograft models. ${ }^{*} \mathrm{P}<0.05,{ }^{* *} \mathrm{P}<0.01,{ }^{* * * *} \mathrm{P}<0.001$.

mean). Student's two-tailed t-test was used for comparison in pairs. The differences between groups ( $>2$ groups) were analyzed with one way analysis of variance (ANOVA) and Tukey's multiple comparison test using GraphPad Prism software version 5.01 (GraphPad Software, Inc., La Jolla, CA, USA). Comparisons without significance are indicated as 'NS'. A P-value $<0.05$ is considered to indicate statistically significant difference $\left({ }^{*} \mathrm{P}<0.05,{ }^{* *} \mathrm{P}<0.01,{ }^{* * *} \mathrm{P}<0.001\right.$ as indicated in the figures). Kaplan-Meier analysis was performed for tumour xenograft models in different treatment arms, with the humane endpoint or death of mice as the outcome measure. The difference in median survival between arms was analysed using log-rank test by Prism.

\section{Results}

In vitro activity of BCT-100 in lung adenocarcinoma cell lines. Treatment with BCT-100 induced a dose-dependent anti-proliferative effect in all lung adenocarcinoma cell lines. The $\mathrm{IC}_{50}$ value of H23, H358, HCC827, H1650, H1975, HCC2935 and HCC4006 cells was $13.1 \pm 1.2,12.8 \pm 1.5,19.1 \pm 7.7$, $25.9 \pm 5.0,12.3 \pm 0.5,620 \pm 65$ and $15.9 \pm 5.1 \mathrm{mU} / \mathrm{ml}$ respectively following a 72 -h treatment.

Basal expression of ASS1 and OTC in vitro. The protein expression of ASS1 was relatively higher in H1975 and HCC2935 cells, lower in H1650 and HCC4006 cells and almost undetectable in H23, H358 and HCC827 cells (Fig. 2). All cell lines were OTC negative (data not shown).

Effects of BCT-100 on tumour xenograft growth. BCT-100 (20 mg/kg) promoted tumour growth in H358, H827, H1650 and H1975 xenograft models, but suppressed growth in the HCC4006 xenograft (Fig. 3A). H358 and HCC827 xenografts were cystic tumours, H1650, H1975 and HCC4006 were solid.

Protein expression of ASS1, OTC, ODC1, SRM and SMS after BCT-100 treatment. Alteration in protein expression of key 
B
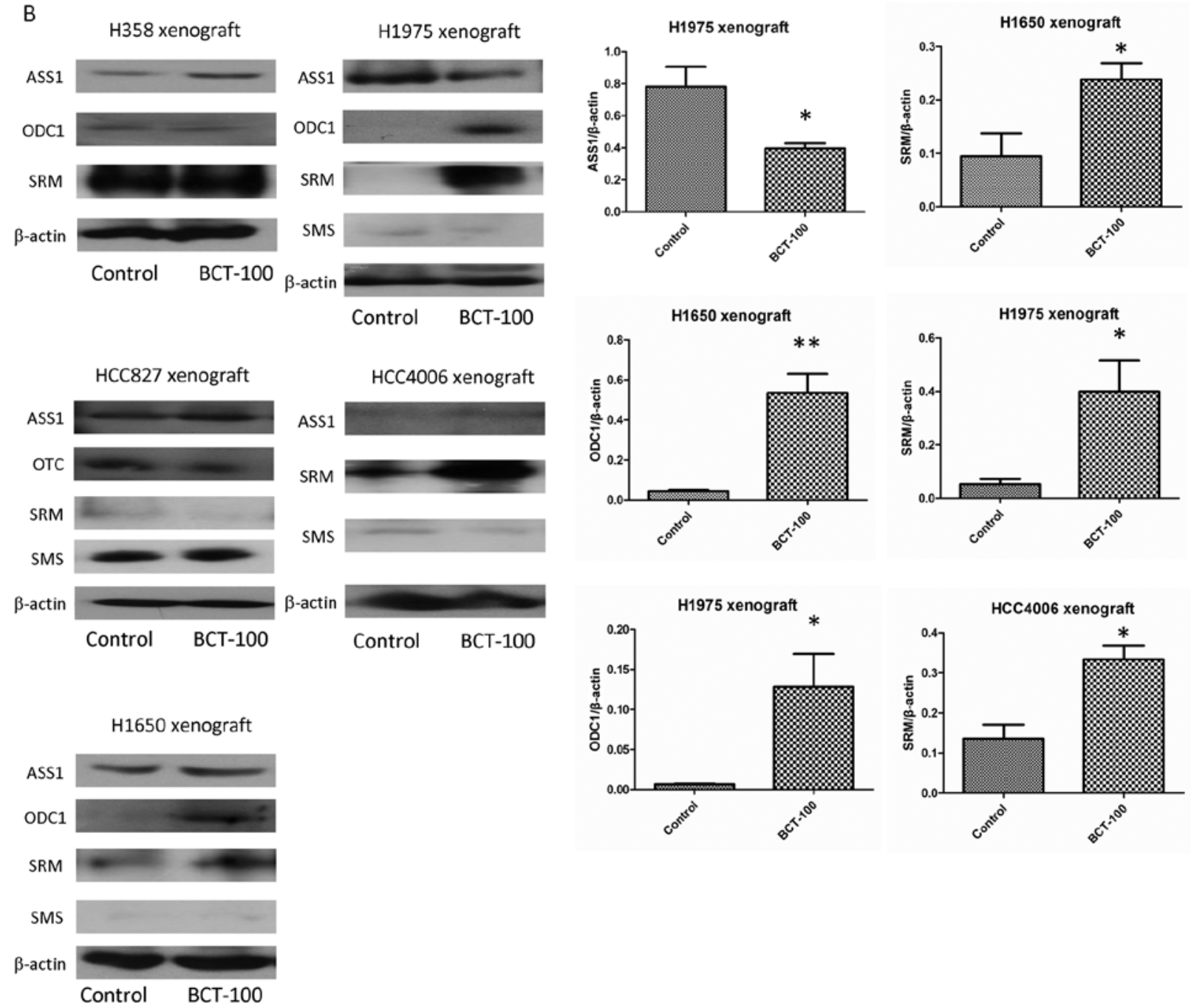

Figure 3. Continued. (B) ASS expression was downregulated in the BCT-100 arm in the H1975 xenograft model. OTC was found and unaltered in HCC 827 xenograft. Upregulation of ODC1 was noted in the BCT-100 arm in both H1650 and H1975 xenograft models. Expression of SRM was unchanged in H358 and HCC827 xenografts but upregulated in H1650, H1975 and HCC4006 xenografts. SMS was unchanged in HCC827, H1650, H1975 and HCC4006 xenografts. ${ }^{*} \mathrm{P}<0.05,{ }^{* *} \mathrm{P}<0.01$.

molecules of arginine metabolism was determined between control and BCT-100 treatment arms. ASS1 was downregulated by BCT-100 in the H1975 xenograft but unaltered in other xenografts. OTC was unchanged in the HCC 827 xenograft and undetectable in others. ODC1 was unaltered in $\mathrm{H} 358$ xenograft but upregulated by BCT-100 in H1650 and H1975 xenografts. ODC1 was undetectable in both control and BCT-100 treatment arms in HCC4006 xenograft (data not shown). SRM was unchanged in $\mathrm{H} 358$ and HCC827 xenografts but upregulated in H1650, H1975 and HCC4006 xenografts. SMS was unaltered in HCC827, H1650, H1975 and HCC4006 xenografts (Fig. 3B).

Effects of combined DFMO and BCT-100 on xenograft models. Relative tumour size increased with BCT-100 treatment in $\mathrm{H} 1650$ and $\mathrm{H} 1975$ xenografts and decreased with BCT-100 in HCC4006 xenograft as described above. There was no significant difference in tumour size between DFMO and control arms in any xenograft model. Combination treatment with DFMO/BCT-100 significantly suppressed tumour growth in H1650 and H1975 xenograft models, in contrast to enhanced tumour growth evident with BCT-100 alone. The effect of DFMO/BCT-100 and BCT-100 remained similar in the HCC4006 xenograft model (Fig. 4A).

Increased median survival with DFMO/BCT-100 in xenograft models. The median survival was increased from 12 days in the control arm to 24 and 25 days in the DFMO/BCT-100 treatment arm $(\mathrm{P}<0.01)$ in $\mathrm{H} 1650$ and $\mathrm{H} 1975$ xenograft models respectively (Fig. 4B).

Intratumoral penetration of BCT-100. As BCT-100 was tagged with PEG, immunoreactivity to anti-PEG antibody was used to quantify the amount of BCT-100 within tumour xenografts. BCT-100 was significantly accumulated within tumours in BCT-100 and DFMO/BCT-100 treatment arms in all three xenograft models (Fig. 5A).

Arginine depletion by DFMO and/or BCT-100 in vivo. Serum arginine concentration and intratumoral arginine content in both BCT-100 and DFMO/BCT-100 treatment arms were significantly decreased in all xenograft models (Fig. 5B). 
A

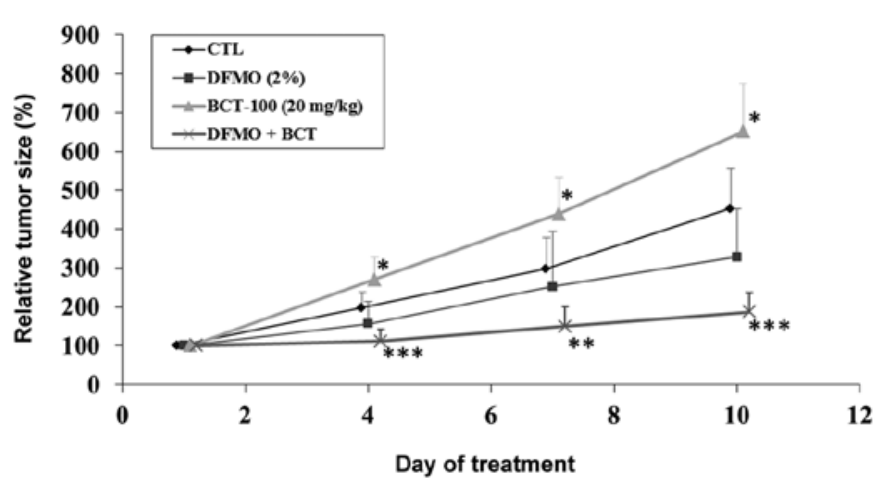

H1975 xenograft

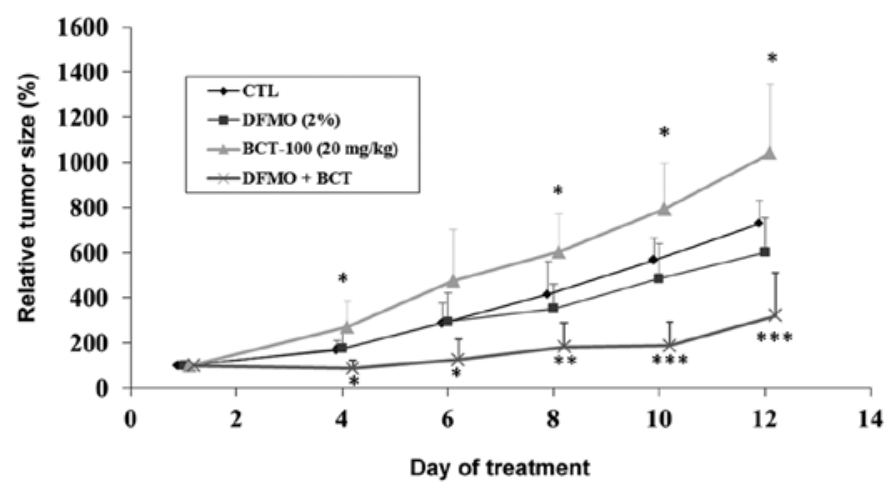

HCC4006 xenograft

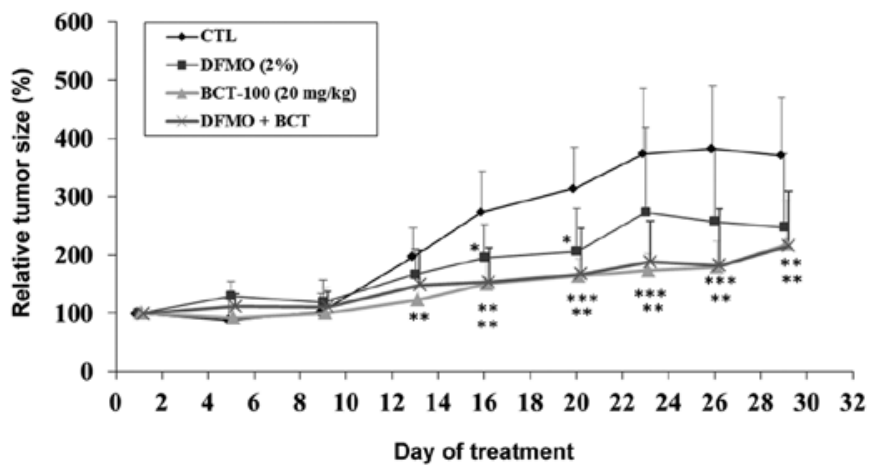

B
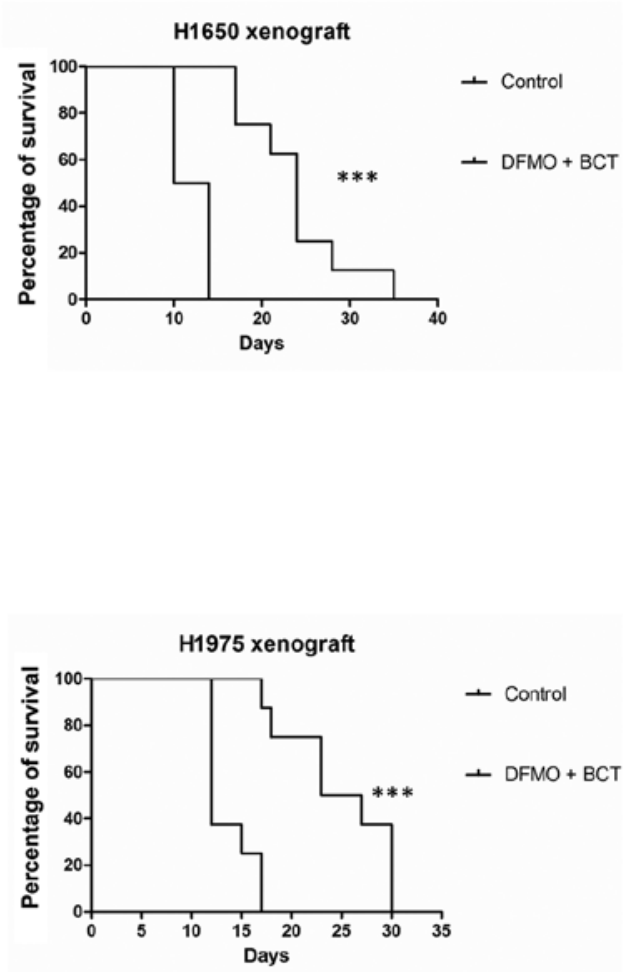

Figure 4. Combination effect of DFMO with BCT-100 in H1650, H1975 and HCC4006 xenograft models. (A) Combination of DFMO with BCT-100 suppressed tumour growth in both H1650 and H1975 xenograft models. The effect of BCT-100 and DFMO/BCT-100 in HCC4006 xenograft model was similar. (B) DFMO/BCT-100 treatment increased median survival in both $\mathrm{H} 1650$ and $\mathrm{H} 1975$ xenograft models. ${ }^{*} \mathrm{P}<0.05,{ }^{* *} \mathrm{P}<0.01,{ }^{* * *} \mathrm{P}<0.001$.

The serum arginine was equally depleted in BCT-100 alone or DFMO/BCT-100 combination across different xenografts. Except for the H1975 xenograft, the DFMO/BCT-100 treatment arm and BCT-100 arm decreased intratumoral arginine to comparable levels (Fig. 5B).

Putrescine and spermidine level in DFMO and/or BCT-100 treatment arms in vivo. Putrescine was decreased in DFMO, BCT-100 and DFMO/BCT-100 groups in all xenograft models except for H1975 xenograft in the BCT-100 arm (Fig. 6A). Decrease in putrescine level in BCT-100 arms was possibly due to putrescine consumption with enhanced tumour growth. Conversely, DFMO declined putrescine level in $\mathrm{DFMO} \pm \mathrm{BCT}-100$ arms resulting from inhibition of ODC1, i.e. decreased putrescine production.

Spermidine level was significantly reduced in DFMO/BCT-100 arm only in H1650 and H1975 xenografts, but remained unaltered in HCC4006 xenograft (Fig. 6B). Spermidine level may be a more important marker than putrescine as an indicator of efficacy in DFMO/BCT-100 combination.

Apoptosis induced by BCT-100 in xenograft models. TUNEL-positive DNA strand breaks and nuclei were stained 
A

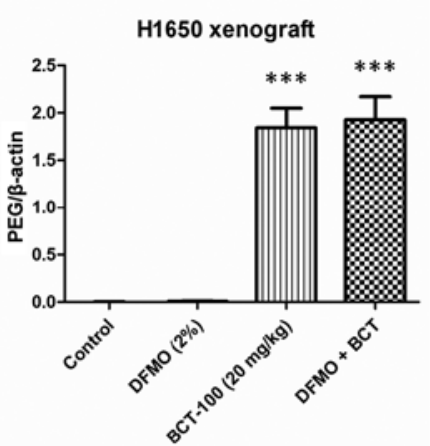

B
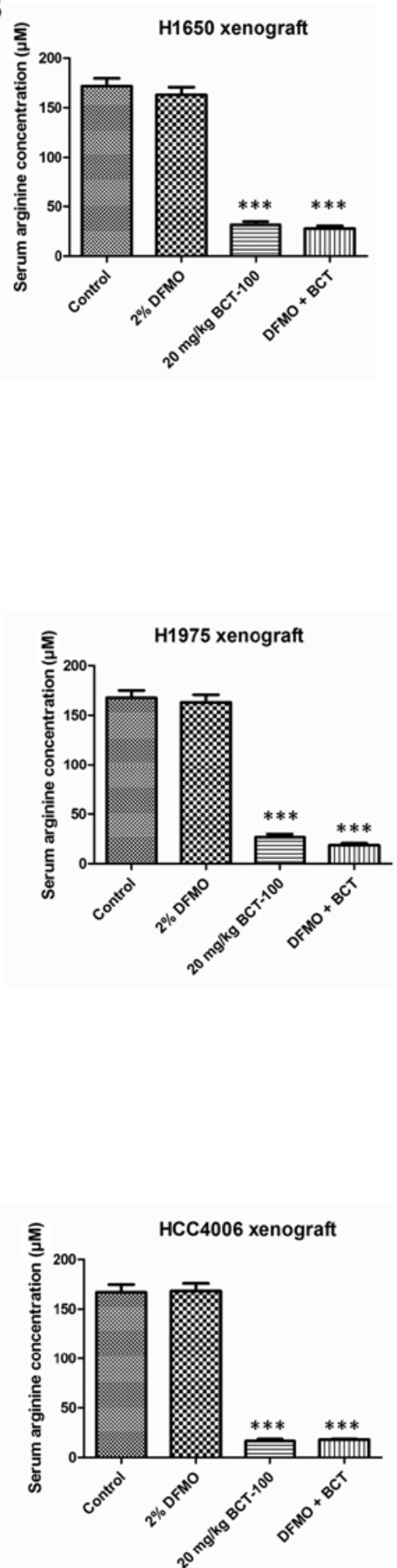
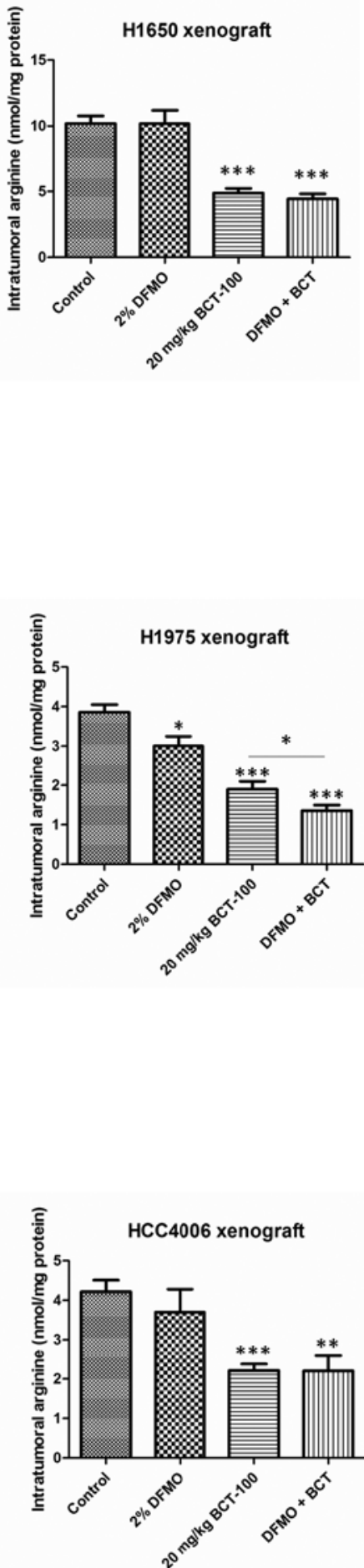
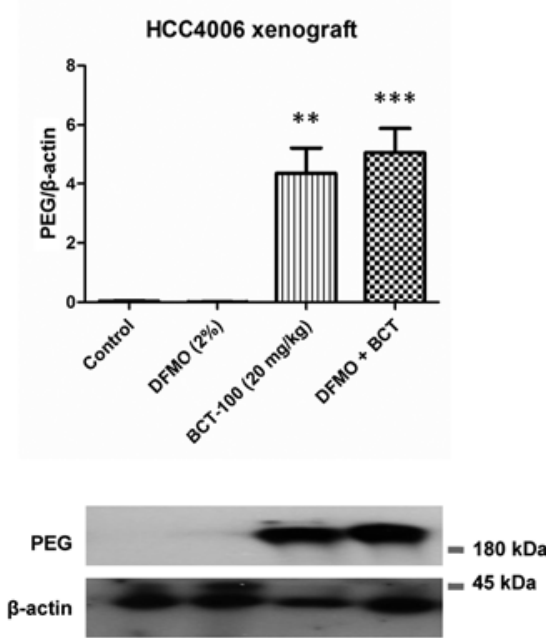

Figure 5. Uptake of PEG-BCT-100 into tumour xenografts as well as serum and intratumoural arginine levels in xenograft models. (A) PEG-BCT-100 was observed in the xenograft samples of BCT and DFMO/BCT-100 arms in all xenograft models. (B) BCT-100 decreased serum arginine concentration and intratumoral arginine level in BCT and DFMO/BCT-100 groups in all xenograft models. Significant difference was noted between BCT and DFMO/BCT-100 group in $\mathrm{H} 1975$ xenograft $\left({ }^{*} \mathrm{P}<0.05,{ }^{* *} \mathrm{P}<0.01,{ }^{* * * *} \mathrm{P}<0.001\right)$.

green and blue, respectively. Apoptotic cells (TUNEL and DAPI-positive) were indicated with double positive staining (Fig. 7). In the H1650 xenograft, the apoptotic signal remained low in the control and DFMO groups, slightly increased in the BCT-100 arm and was further elevated in the DFMO/BCT-100 combination group (Fig. 7A). In the 
A

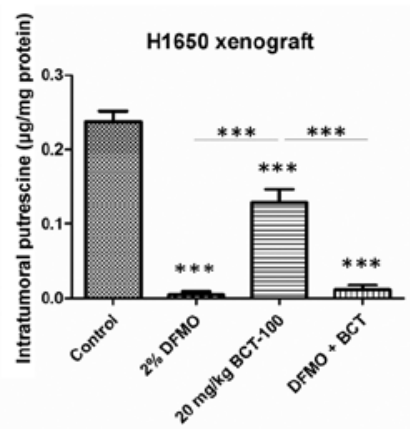

B
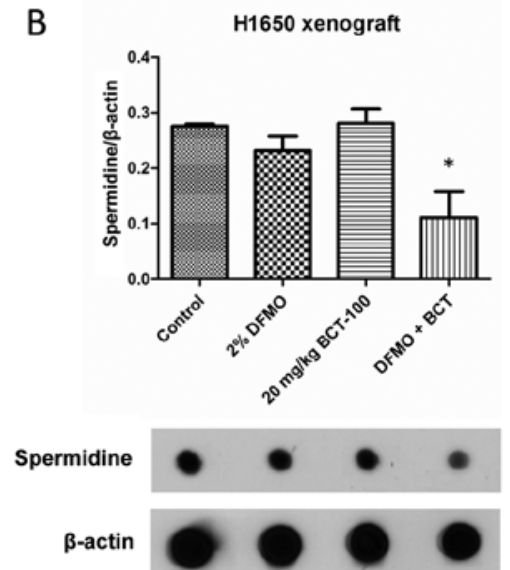
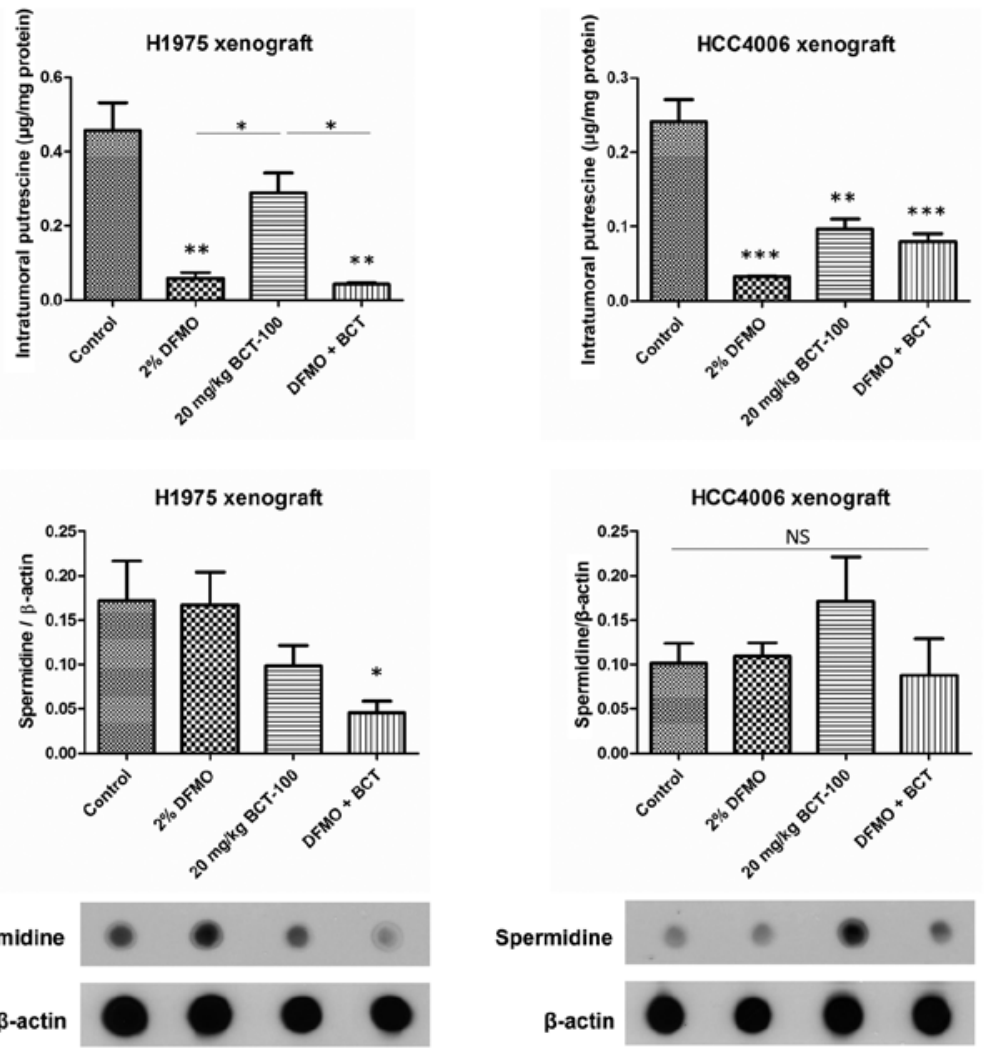
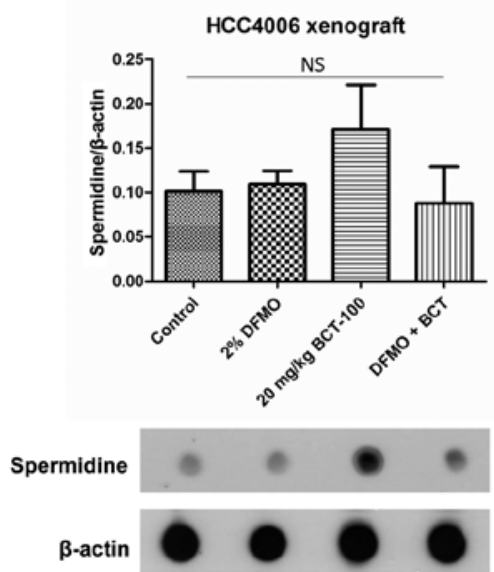

Figure 6. Decreased intratumoral putrescine and spermidine level upon DFMO and/or BCT-100 treatment. (A) Intratumoral putrescine was decreased in all DFMO and/or BCT-100 treatment groups except the BCT-100 treatment arm in the H1975 xenograft model. (B) Intratumoral spermidine level was reduced by DMFO/BCT combination arms in $\mathrm{H} 1650$ and $\mathrm{H} 1975$ xenograft models. ${ }^{*} \mathrm{P}<0.05,{ }^{* *} \mathrm{P}<0.01,{ }^{* * *} \mathrm{P}<0.001$; NS, not significant.

H1975 xenograft, the level of apoptosis was maintained at a low level in control, DFMO and BCT-100 groups, but significantly enhanced in the DFMO/BCT-100 combination arm (Fig. 7B). In the HCC4006 xenograft, apoptotic signal was noted in DFMO, BCT-100 and DFMO/BCT-100 arms but not in the control group (Fig. 7C). The percentage of cells that underwent apoptosis is displayed in Fig. 7D. Furthermore, the upregulation of cleaved PARP (cPARP) was observed in the DFMO/BCT-100 treatment arm in both H1650 and H1975 xenograft models (Fig. 7E), but not in the HCC4006 xenograft (data not shown). Downregulation of survivin, serving as an anti-apoptotic factor, was also observed in DFMO/BCT-100 treatment group in H1975 xenograft (Fig. 7E).

\section{Discussion}

BCT-100 demonstrated anti-proliferative effects in vitro, however, demonstrated a paradoxical tumour promoting effect in most of our in vivo xenograft models. ODC1 was upregulated by BCT-100 treatment in H1650 and H1975 xenograft models. We postulated that ornithine produced from arginine by BCT-100 was converted to polyamines by upregulated ODC1, thus leading to stimulation of tumour growth. The combination of an ODC1 inhibitor (DFMO) with BCT-100 restored the tumour suppressive effects of BCT-100 in the H1650 and H1975 xenograft models with an increased median survival, mediated by decreasing serum and intratumoral arginine levels as well as intratumural putrescine and spermidine levels. Furthermore, apoptosis was enhanced by
DFMO/BCT-100 in H1650 and H1975 xenografts, in keeping with the observed antitumoral effects. Nonetheless there was no beneficial or detrimental effect in the HCC4006 xenograft model (whereby ODC1 was not induced by BCT-100) when DFMO and BCT-100 were combined.

Based on the updated GLOBOCAN project of the World Health Organization in 2012, lung cancer is the top cancer killer (http://globocan.iarc.fr/). The incidence and mortality rates of lung cancer were 16.7 and $23.2 \%$, respectively. Lung cancer can be classified as non-small cell lung carcinoma or small cell lung carcinoma. Eighty-five percent of lung cancer cases are NSCLC and adenocarcinoma is the major subtype. Systemic chemotherapy remains the cornerstone treatment for NSCLC with only a modest survival benefit. Although targeted therapies (e.g. against EGFR) have been developed for lung cancer patients with different types of mutations, development of acquired drug resistance around one year following targeted therapy is practically unavoidable (15). As such, novel treatment for NSCLC is highly desired.

Amino acid depletion is a potentially promising approach of anticancer therapy, akin to the use of L-asparaginase in the treatment of acute leukaemia. Arginine is an essential amino acid for cancer cells that are incapable of replenishing their arginine store, but not normal cells. Arginine deiminase (ADI) and arginase have been used to deplete arginine in in vivo and clinical studies. ADI induces an anticancer effect on ASS1-deficient cancers including those of the head and neck, lymphoma, pancreas, breast and small cell lung carcinoma (16) by cell cycle arrest and apoptosis. Arginase (BCT-100) also 


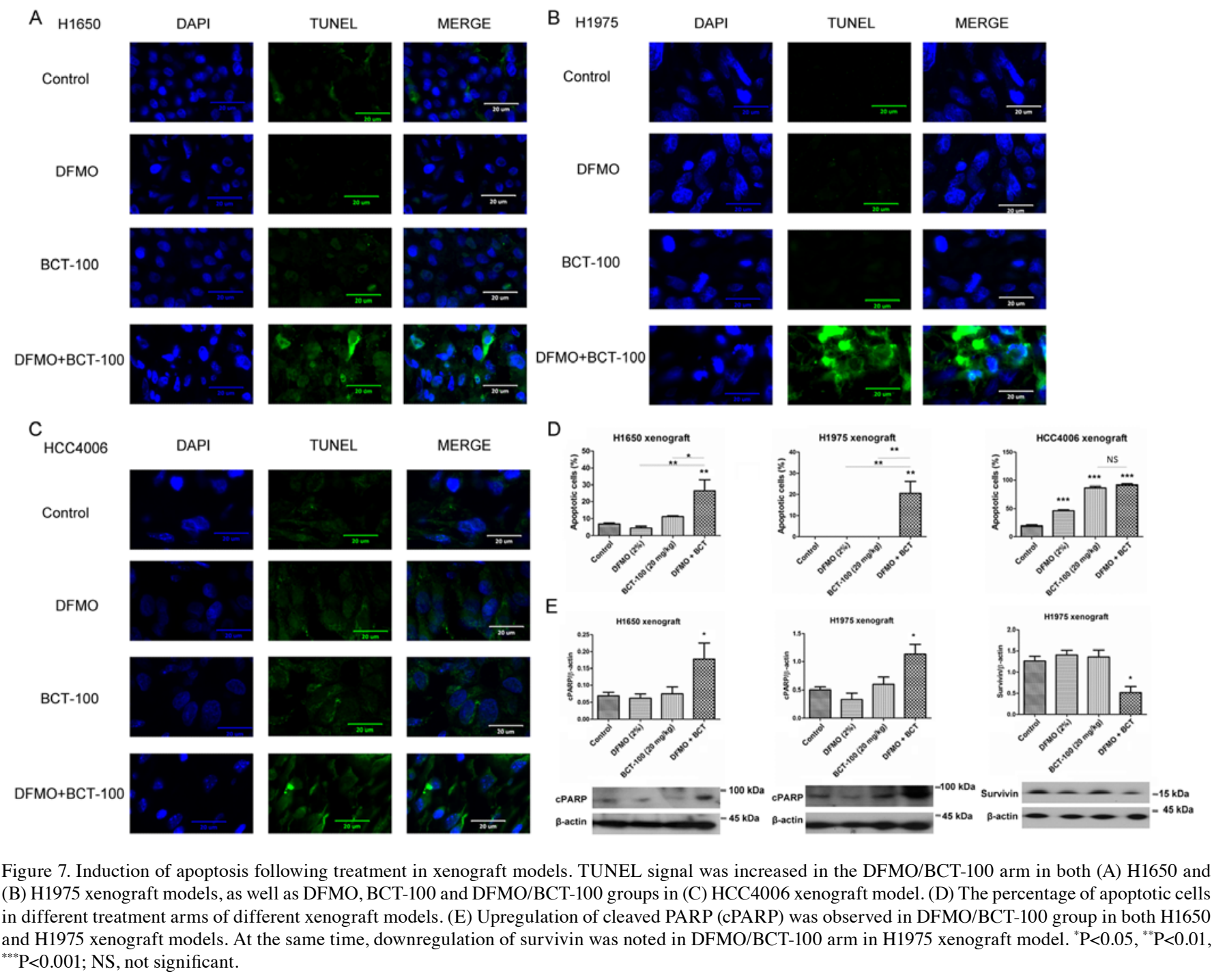

displays anticancer activity in melanoma (8) and human hepatoma (2) via apoptosis, as well as cell cycle arrest in melanoma (8), HCC (9) and acute myeloid leukaemia (17).

BCT-100 reduced cell viability in a panel of lung adenocarcinoma cell lines but promoted tumour growth in most lung adenocarcinoma xenograft models. Concurrently, ODC1 was upregulated when treated with BCT-100. For tumour xenografts (H1650 and H1975) that did not express OTC, ornithine accumulated upon exposure to BCT-100. Of note, ODC1 is an inducible enzyme. An increased ornithine level (substrate of ODC1) has been shown to induce ODC1 expression (18). Conversely, ODC1 can be upregulated by translational and transcriptional mechanisms via PI3-kinase/Akt/mTOR and Ras/Raf/MEK/Erk/c-myc pathways respectively (19). In addition, ODC1 can be induced during hypoxia which increased polyamines production and promoted cell survival in different cell lines. Notably, apoptosis was induced when depleting polyamines during hypoxic stress (20). BCT-100 depleted arginine to ornithine and may have been responsible for upregulation of ODC1 expression in H1650 and H1975 solid tumour xenografts. Notably, ODC1 expression was not induced with in vitro exposure to BCT-100 (data not shown), which was postulated to be due to the limited amount of arginine in in vitro culture

medium, but relatively abundant source of arginine from food in vivo. The excessive arginine in vivo was then converted to ornithine by BCT-100 which induced ODC1 expression.

Ornithine was then converted to polyamines by ODC1,SRM and SMS. Polyamines are known to promote cell proliferation and tumour growth (21). In addition, SRM is also an inducible enzyme by its own substrate (putrescine) (22). BCT-100 not only upregulated ODC1, but also SRM, and this may speed up the conversion of putrescine to spermidine. Increased polyamines production may counteract or even overwhelm the tumour suppression effect of arginase treatment. Nonetheless, conversion of polyamines from ornithine could be inhibited by ODC1 inhibitors (e.g. $\alpha$-difluoromethylornithine or DFMO). In order to prevent the catastrophic effect due to increased polyamines synthesis, we proposed that a combination of ODC1 inhibitor with BCT-100 could restore the therapeutic function of BCT-100 in lung adenocarcinoma.

DFMO is a specific ODC1 inhibitor that has been used in a phase III clinical trial for chemoprevention of sporadic colorectal adenomas (23). It has been shown to significantly decrease putrescine and spermidine level, but not spermine level, in both human colon cancer (24) and xenograft mouse models of skin squamous cell carcinoma (25). Nonetheless 
there was no direct tumour-suppressive effect of DFMO in our lung adenocarcinoma xenograft models. In the present study, the putrescine level was decreased in the BCT-100 treatment arms, yet DFMO inhibited ODC1 that also suppressed putrescine production. In our study, BCT-100 did not increase the spermidine level that was probably used up for protein synthesis and cell proliferation (26). As previously reported in the literature, activation of ODC1 often decreases the level of polyamines (26) and is consistent with our findings.

Depletion of arginine (BCT-100 treatment) and polyamine (DFMO treatment) suppressed tumour growth in solid tumour xenograft models with inducible ODC1. At the same time, the tumour-suppressive effect of BCT-100 was not hampered by DFMO treatment in a solid tumour xenograft model without ODC1 induction. It is of note that high basal ODC1 expression has been found in liver and colon cancers (27), MYCN-amplified neuroblastoma (28) and leukaemia (29). Whether induction of ODC1 expression by BCT-100 occurs in cancers other than lung adenocarcinoma need to be elucidated. Theoretically, upregulation of polyamines by high basal or inducible ODC1 expression can be potentially aborted by DFMO. Our findings indicated that a combination of DFMO with BCT-100 can abrogate a compensatory mechanism (via production of polyamines) that hinders the therapeutic effects of BCT-100 in lung adenocarcinoma.

BCT-100 has been tested in different cancer models, including hepatocellular carcinoma, acute myeloid leukemia, acute lymphoblastic leukemia, glioblastoma, melanoma, prostate and pancreatic cancer and mesothelioma. BCT-100 induced apoptosis and/or cell cycle arrest in most cases (30), while promoted tumour growth paradoxically in the present study. A combination of DFMO and BCT-100 re-directed cancer cells to apoptosis. However, cell cycle arrest was not observed as there was no alteration in the expression of different cyclins and cyclin-dependent kinases (data not shown).

A recent early phase clinical trial of BCT-100 treatment (1,600 U/kg/week) in HCC was completed in our institution. A significant increase in progression-free survival and overall survival was observed in patients with adequate arginine depletion for $>2$ months. The adverse effects associated with BCT-100 treatment were mild and included loss of appetite, pain, vomiting, constipation, insomnia, fatigue and nausea (10). Further clinical exploration of BCT-100 in the treatment of $\mathrm{HCC}$ and acute leukaemia are ongoing.

In a phase III chemoprevention trial for sporadic colorectal adenomas, DFMO $500 \mathrm{mg}$ daily in combination with sulindac $150 \mathrm{mg}$ daily for 3 years was associated with few reported adverse events. There was no significant difference $(\mathrm{P}>0.05)$ between placebo and treatment groups in grade 3 or above adverse events, frequency of overnight hospitalizations, cardiovascular events, deaths, hearing problems and gastrointestinal events (23).

In conclusion, an ODC1-inhibitor (e.g. DFMO) should be used in conjunction with pegylated arginase (BCT-100) in the treatment of lung adenocarcinoma.

\section{Acknowledgements}

Not applicable.

\section{Funding}

No funding was received.

\section{Availability of data and materials}

The datasets used during the present study are available from the corresponding author upon reasonable request.

\section{Authors' contributions}

SKL, KPU, YYL, SX, PNMC and JCMH substantially contributed to the conception or design of the work, to the acquisition, analysis and interpretation of data. SKL and JCMH drafted the work or revised it critically for important intellectual content. SKL and JCMH approved the final version to be published. All authors agree to be accountable for all aspects of the work in ensuring that questions related to the accuracy or integrity of any part of the work are appropriately investigated and resolved.

\section{Ethics approval and consent to participate}

The study protocol was approved by the institutional Animal Ethics Committee of The University of Hong Kong (approval ref. no. CULATR 3781-15) and standard humane endpoints for animal research were applied in compliance with the instructions of U.S. Public Health Service (policy on humane care and use of laboratory animals).

\section{Patient consent for publication}

Not applicable.

\section{Competing interests}

YYL and SX report no potential conflict of interest. PNC is the Chief Executive Officer of Bio-Cancer Treatment International Limited and holds stocks or shares in Bio-Cancer Treatment International Limited.KPU was a Scientific Officer at Bio-Cancer Treatment International Limited. SKL, KPU, PNMC and JCH hold patents relating to the content of the manuscript.

\section{References}

1. Delage B, Fennell DA, Nicholson L, McNeish I, Lemoine NR, Crook T and Szlosarek PW: Arginine deprivation and argininosuccinate synthetase expression in the treatment of cancer. Int J Cancer 126: 2762-2772, 2010.

2. Chow AK, Ng L, Sing Li H, Cheng CW, Lam CS, Yau TC, Cheng PN, Fan ST, Poon RT and Pang RW: Anti-tumor efficacy of a recombinant human arginase in human hepatocellular carcinoma. Curr Cancer Drug Targets 12: 1233-1243, 2012.

3. Feun L and Savaraj N: Pegylated arginine deiminase: A novel anticancer enzyme agent. Exp Opin Invest Drugs 15: 815-822, 2006.

4. Thomas T and Thomas TJ: Polyamines in cell growth and cell death: Molecular mechanisms and therapeutic applications. Cell Mol Life Sci 58: 244-258, 2001.

5. Tanios R, Bekdash A, Kassab E, Stone E, Georgiou G, Frankel AE and Abi-Habib RJ: Human recombinant arginase I(Co)-PEG5000 [HuArgI(Co)-PEG5000]-induced arginine depletion is selectively cytotoxic to human acute myeloid leukemia cells. Leuk Res 37: 1565-1571, 2013. 
6. Cheng PN, Lam TL, Lam WM, Tsui SM, Cheng AW, Lo WH and Leung YC: Pegylated recombinant human arginase $\left(\right.$ rhArg-peg $\left.{ }_{5000 \mathrm{mw}}\right)$ inhibits the in vitro and in vivo proliferation of human hepatocellular carcinoma through arginine depletion. Cancer Res 67: 309-317, 2007.

7. Lam SK, Li YY, Xu S, Leung LL, U KP, Zheng YF, Cheng PN and Ho JC: Growth suppressive effect of pegylated arginase in malignant pleural mesothelioma xenografts. Respir Res 18: 80, 2017.

8. Lam TL, Wong GK, Chow HY, Chong HC, Chow TL, Kwok SY, Cheng PN, Wheatley DN, Lo WH and Leung YC: Recombinant human arginase inhibits the in vitro and in vivo proliferation of human melanoma by inducing cell cycle arrest and apoptosis. Pigment Cell Melanoma Res 24: 366-376, 2011.

9. Lam TL, Wong GK, Chong HC, Cheng PN, Choi SC, Chow TL, Kwok SY, Poon RT, Wheatley DN, Lo WH, et al: Recombinant human arginase inhibits proliferation of human hepatocellular carcinoma by inducing cell cycle arrest. Cancer Lett 277: 91-100, 2009.

10. Yau T, Cheng PN, Chan P, Chen L, Yuen J, Pang R, Fan ST, Wheatley DN and Poon RT: Preliminary efficacy, safety, pharmacokinetics, pharmacodynamics and quality of life study of pegylated recombinant human arginase 1 in patients with advanced hepatocellular carcinoma. Invest New Drugs 33: 496-504, 2015

11. Lam SK, Li YY, Zheng CY and Ho JC: Downregulation of thymidylate synthase and $\mathrm{E} 2 \mathrm{~F} 1$ by arsenic trioxide in mesothelioma. Int J Oncol 46: 113-122, 2015.

12. Li YY,Lam SK, Mak JC,Zheng CY and Ho JC: Erlotinib-induced autophagy in epidermal growth factor receptor mutated non-small cell lung cancer. Lung Cancer 81: 354-361, 2013.

13. Brenner T, Fleming TH, Spranz D, Schemmer P, Bruckner T, Uhle F, Martin EO, Weigand MA and Hofer S: Reactive metabolites and AGE-RAGE-mediated inflammation in patients following liver transplantation. Mediators Inflamm 2013: 501430, 2013.

14. Nicoletti R, Venza I, Ceci G, Visalli M, Teti D and Reibaldi A: Vitreous polyamines spermidine, putrescine, and spermine in human proliferative disorders of the retina. $\mathrm{Br} \mathrm{J}$ Ophthalmol 87 1038-1042, 2003.

15. Ho JC, Tam TC and Lam SK: Salvage therapy beyond targeted therapy in lung adenocarcinoma. Semin Respir Crit Care Med 34 837-844, 2013.

16. Qiu F, Huang J and Sui M: Targeting arginine metabolism pathway to treat arginine-dependent cancers. Cancer Lett 364: $1-7,2015$.

17. Mussai F, Egan S, Higginbotham-Jones J, Perry T, Beggs A, Odintsova E, Loke J, Pratt G, U KP, Lo A, et al: Arginine dependence of acute myeloid leukemia blast proliferation: A novel therapeutic target. Blood 125: 2386-2396, 2015.

18. Majumdar R, Shao L, Minocha R, Long S and Minocha SC: Ornithine: The overlooked molecule in the regulation of polyamine metabolism. Plant Cell Physiol 54: 990-1004, 2013.
19. Shantz LM and Levin VA: Regulation of ornithine decarboxylase during oncogenic transformation: Mechanisms and therapeutic potential. Amino Acids 33: 213-223, 2007.

20. Svensson KJ, Welch JE, Kucharzewska P, Bengtson P, Bjurberg M, Påhlman S, Ten Dam GB, Persson L and Belting M: Hypoxia-mediated induction of the polyamine system provides opportunities for tumor growth inhibition by combined targeting of vascular endothelial growth factor and ornithine decarboxylase. Cancer Res 68: 9291-9301, 2008.

21. Miller-Fleming L, Olin-Sandoval V, Campbell K and Ralser M Remaining mysteries of molecular biology: The role of polyamines in the cell. J Mol Biol 427: 3389-3406, 2015.

22. Russell DH and Snyder SH: Amine synthesis in regenerating rat liver: Extremely rapid turnover of ornithine decarboxylase. Mol Pharmacol 5: 253-262, 1969.

23. Meyskens FL Jr, McLaren CE, Pelot D, Fujikawa-Brooks S, Carpenter PM, Hawk E, Kelloff G, Lawson MJ, Kidao J, McCracken J, et al: Difluoromethylornithine plus sulindac for the prevention of sporadic colorectal adenomas: A randomized placebo-controlled, double-blind trial. Cancer Prev Res (Phila) 1: 32-38, 2008.

24. Mackenzie GG, Ouyang N, Xie G, Vrankova K, Huang L, Sun Y, Komninou D, Kopelovich L and Rigas B: Phospho-sulindac (OXT-328) combined with difluoromethylornithine prevents colon cancer in mice. Cancer Prev Res 4: 1052-1060, 2011.

25. Chen $\mathrm{Y}, \mathrm{Hu}$ J, Boorman D, Klein-Szanto $\mathrm{A}$ and O'Brien TG: Therapy of murine squamous cell carcinomas with 2-difluoromethylornithine. J Carcinog 3: 10, 2004.

26. Schipper RG, Penning LC and Verhofstad AA: Involvement of polyamines in apoptosis. Facts and controversies: Effectors or protectors? Semin Cancer Biol 10: 55-68, 2000.

27. Tomasi ML, Ryoo M, Skay A, Tomasi I, Giordano P, Mato JM and $\mathrm{Lu}$ SC: Polyamine and methionine adenosyltransferase 2A crosstalk in human colon and liver cancer. Exp Cell Res 319: 1902-1911, 2013.

28. Rounbehler RJ, Li W, Hall MA, Yang C, Fallahi M and Cleveland JL: Targeting ornithine decarboxylase impairs development of $M Y C N$-amplified neuroblastoma. Cancer Res 69: 547-553, 2009.

29. Wang MF, Liao YF, Hung YC, Lin CL, Hour TC, Lue KH, Hung HC and Liu GY: Hydroxydibenzoylmethane induces apoptosis through repressing ornithine decarboxylase in human promyelocytic leukemia HL-60 cells. Exp Mol Med 43: 189-196, 2011.

30. Fung MKL and Chan GC: Drug-induced amino acid deprivation as strategy for cancer therapy. J Hematol Oncol 10: 144, 2017.

This work is licensed under a Creative Commons Attribution-NonCommercial-NoDerivatives 4.0 International (CC BY-NC-ND 4.0) License. 